\title{
Dynamical mass generation for fermions in quenched Quantum Electrodynamics at finite temperature
}

\author{
Alejandro Ayala ${ }^{\dagger}$, Adnan Bashir ${ }^{\ddagger}$ \\ $\dagger$ Instituto de Ciencias Nucleares, Universidad Nacional Autónoma de México, \\ Apartado Postal 70-543, México Distrito Federal 04510, México \\ ${ }^{\ddagger}$ Instituto de Física y Matemáticas, Universidad Michoacana de San Nicolás de Hidalgo, \\ Apartado Postal 2-82, Morelia, Michoacán 58040, México
}

\begin{abstract}
We study the dynamical generation of masses for fundamental fermions in quenched quantum electrodynamics (qQED) at finite temperature in the bare vertex approximation, using SchwingerDyson equations (SDE). Motivated by perturbation theory, a further simplification is introduced by taking the wave function renormalization to be unity. In the zeroth mode approximation, the SDE for the fermion propagator resembles QED in 2+1 dimensions (QED3) at zero temperature with an effective dimensionful coupling $\alpha^{\prime}=\alpha T$. For a fixed temperature, mass is dynamically generated above a certain critical value of this coupling. As expected, raising the temperature restores chiral symmetry and fermions become massless again. We also argue that by summing over the frequency modes and under suitable simplifications, qualitative aspects of the result do not undergo significant changes.
\end{abstract}

PACS numbers: 11.15.Tk, 12.20.-m, 11.10.Wx

\section{INTRODUCTION}

The perturbative treatment of gauge field theories at zero temperature is valid only for weak coupling, which fails to explain certain non-perturbative aspects of these theories such as dynamical mass generation for fermions. Schwinger-Dyson equations (SDEs) provide us with a natural platform to study this phenomenon because their derivation makes no assumption about the strength of the interaction. In quantum electrodynamics (QED) at zero temperature, it is well known that above a certain critical value of the coupling, fermions can acquire masses through self interaction without the necessity of a non zero bare mass. This fact is in marked contrast to perturbation theory which prohibits the appearance of masses in this fashion as long as the bare mass is zero even if we did an all order resummation using renormalization group equations. In a thermal background, temperature is another parameter which comes into play and we expect criticality in it too, triggering mass generation below a certain temperature and restoring chiral symmetry if we go above this value. The relevant SDE to investigate such a behavior is that of the fermion propagator.

SDEs are an infinite tower of coupled equations relating various Green's functions to each other. The twopoint Green's function of the fermion propagator is coupled to all higher order Green's functions. Therefore, a non-perturbative truncation is essential to convert it into a solvable form. In quenched quantum electrodynamics (qQED), a favorite starting point is to make an ansatz for the fermion-photon vertex and then study the fermion propagator equation in its decoupled form. Inspired by some key features of gauge theories such as WardGreen-Takasahi identities, Landau-Khalatnikov-Fradkin transformations, multiplicative renormalizability of the fermion propagator, etc., significant advances have been made in the hunt for a reliable vertex ansatz at zero tem- perature and the corresponding study of dynamical chiral symmetry breaking. However, for finite temperature, this venue has been much less explored, partially because of the richer tensor structure of the propagator and the vertex involved [1, 2, 3, 4, 5, 6, 7]. The case of three dimensional QED (QED3) at finite temperature has been studied in relatively more detail in the bare vertex approximation [8, 9, 10, 11, 12, 13, 14].

In this paper, we undertake the study of QED4 in the quenched approximation at finite temperature. We study the corresponding Schwinger-Dyson equation for the fermion propagator in the bare vertex approximation. Under the additional assumptions of setting the wave function renormalization to unity and picking out only the most dominant frequency mode, the theory resembles QED3 at zero temperature (see also [15]). The effective coupling $\alpha T$ is dimensionful. In order to pursue the resemblance of the theory with QED3, we can take $\alpha^{\prime}=\alpha T$ along with $T$ as independent parameters. The only notable difference is a temperature dependent mass term. The effect of this term is such that keeping the temperature constant, the increase of the coupling beyond a certain critical value makes the fermion to acquire a mass. Conversely, increasing the temperature above a threshold value, we observe chiral symmetry restoration. We also confirm this behavior analytically. Moreover, increasing the value of the coupling, the critical value of the temperature also gets multiplied by the same factor. We must emphasize that the existence of criticality for the coupling and temperature is in contrast to the behavior of QED3 at zero temperature where no criticality exists: if masses are generated for one value of the coupling, they are generated for all other values of the coupling. Although not explicitly considered, the analysis can easily be extended to the case in which $\alpha$ and $T$ are taken as the independent parameters.

The work is organized as follows: In Sec. II we set up 
the SDE for the mass function in $\mathrm{qQED}$ with bare vertex at finite temperature using the imaginary-time formalism, starting from a simplified tensor structure for the fermion propagator. In Sec. IIII we work under the assumption of considering only the contribution from the lowest Matsubara frequency finding a non-trivial numerical solution for the mass function. We study critical behavior of the mass function with the coupling strength as well as with temperature. With further simplifications in the integral equation, we also study this critical behavior analytically, confirming the numerical results. In Sec. IV] we relax the assumption that considered the lowest Matsubara frequency as the single contributing mode and perform the sum over frequencies. We analytically study the resulting integral equation under the same simplifying assumptions as before and argue that, except for slightly different numerical coefficients, the behavior of the mass function appears to be qualitatively the same. We finally summarize and discuss our results in Sec. $\nabla$

\section{THE FERMION PROPAGATOR AND THE MASS FUNCTION}

The Minkowski space Feynman rules for the QED bare fermion propagator, photon propagator and fermionphoton vertex are, respectively,

$$
\begin{aligned}
i S_{F}^{0}(P) & =\frac{i}{P}, \\
D_{0}^{\mu \nu}(Q) & =\frac{i}{Q^{2}}\left[-g^{\mu \nu}+(1-\xi) \frac{Q^{\mu} Q^{\nu}}{Q^{2}}\right], \\
i g \Gamma_{0}^{\mu}(K, P) & =i g \gamma^{\mu},
\end{aligned}
$$

(hereafter we use capital letters to refer to four-vectors, whereas lower case letters are used for their components) where $g$ is the electromagnetic coupling and $\xi$ is the usual covariant gauge parameter. Making use of the above rules, the SDE for the fermion propagator can be written as

$$
\begin{aligned}
S_{F}^{-1}(P) & =\left(S_{F}^{0}\right)^{-1}(P) \\
& -i g^{2} \int \frac{d^{4} K}{(2 \pi)^{4}} \Gamma^{\mu}(K, P) S_{F}(K) \gamma^{\nu} D_{\mu \nu}^{0}(Q),
\end{aligned}
$$

where $Q=K-P, \Gamma^{\nu}(K, P)$ is the full fermion-photon vertex and $S_{F}(P)$ is the full fermion propagator.

At finite temperature, the most general tensor form for the fermion propagator can be obtained by noticing that, in addition to 1 and $\not P$, there are also two other Lorentz structures, namely $\Psi$ and $P X$. Therefore, we can write

$$
S_{F}(P)=\frac{F}{P-b \Psi-c P \Psi-M} .
$$

The Lorentz invariant functions $F, b, c$ and $M$ will in general depend on two Lorentz scalars $P^{2}$ and $U \cdot P$, where $U^{\mu}$ is the four-velocity of the heat bath as seen from a general frame. We can choose these scalars to be

$$
\begin{aligned}
p_{0} & \equiv P^{\mu} U_{\mu} \\
p & \equiv\left[\left(P^{\mu} U_{\mu}\right)^{2}-P^{2}\right]^{1 / 2} .
\end{aligned}
$$

The functions $F$ and $M$ are called the wave function renormalization and mass function, respectively. Notice that for a parity conserving theory such as QED, $c=0$ [16].

For our purposes, we will work in the approximation where $b=0$ and $F=1$. The justification for this approximation stems from the fact that perturbatively $F=1+\mathcal{O}(\alpha)$ and $b=\mathcal{O}(\alpha)$. If $\alpha$ is small, we naturally expect $F \approx 1$ and $b \approx 0$. Though it is well known that at zero temperature, inclusion of $F$ is essential to recover the gauge invariance of the results such as the generated fermion mass 17], it is also known that when ignoring $F$, the qualitative results, such as the shape of the fermion mass as a function of the coupling strength, are not changed. At finite temperature, we can expect that $F$ and $b$ play a similar role for the restoration of the gauge invariance 10]. Nevertheless, to keep the discussion as simple as possible, here we will write the fermion propagator as

$$
S_{F}(P)=\frac{1}{P-M(P)},
$$

ignoring $F$ and $b$ in Eq. (3).

Taking the trace of Eq. (2), making use of Eqs. (11) and (5), and replacing the full vertex by its bare counterpart, the equation for the mass function can be written in Minkowski space as

$$
M(P)=-i \frac{(3+\xi) \alpha}{4 \pi^{3}} \int d^{4} K \frac{M(K)}{K^{2}-M^{2}(K)} \frac{1}{Q^{2}} .
$$

We work explicitly in the imaginary-time formulation of thermal field theory, taking $K=\left(i \omega_{n}, \mathbf{k}\right), P=(i \omega, \mathbf{p})$ where $\omega_{n}=(2 n+1) \pi T$ and $\omega=(2 m+1) \pi T$ are discrete fermion frequencies, with $T$ being the temperature and $n, m$ integers. In this formalism, Eq. (6) becomes

$$
\begin{aligned}
M(P) & =\frac{(3+\xi) \alpha}{2 \pi^{2}} T \sum_{n} \int d^{3} k\left\{\frac{M(K)}{\left[\omega_{n}^{2}+\mathbf{k}^{2}+M^{2}(K)\right]}\right. \\
& \left.\times \frac{1}{\left[\left(\omega_{n}-\omega\right)^{2}+|\mathbf{k}-\mathbf{p}|^{2}\right]}\right\} .
\end{aligned}
$$

\section{ZEROTH MODE APPROXIMATION}

\section{A. The full equation}

A trivial solution to Eq. (7) is $M(P)=0$, which corresponds to the usual perturbative solution. However, we are interested in a non-trivial solution. To pursue it, as a first step we make the approximation where we take only 


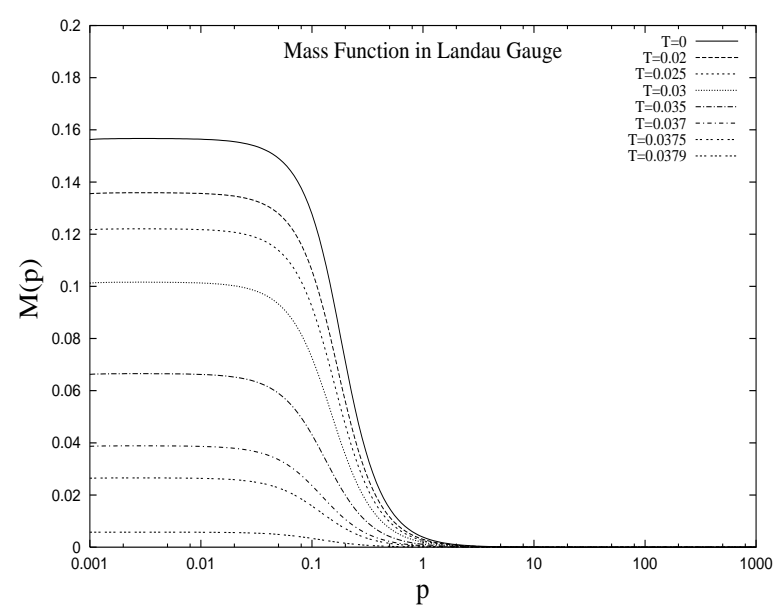

FIG. 1: The mass function $M(p)$ for various values of temperature and $\alpha^{\prime}=1 / 4 \pi$ in the Landau gauge.

the contribution of the zeroth mode, i.e., $n=0$, in the sum over frequencies in Eq. (7) and correspondingly take the same value for $\omega$. We relax this approximation in the following section. It appears natural to take $\alpha T=\alpha^{\prime}$ and $T$ as independent variables. After carrying out the angular integration, Eq. (7) becomes

$M(p)=\frac{(3+\xi) \alpha^{\prime}}{2 \pi p} \int_{0}^{\infty} \frac{d k k M(k)}{\pi^{2} T^{2}+k^{2}+M^{2}(k)} \ln \left|\frac{k+p}{k-p}\right|$,

where $p$ and $k$ are the magnitudes of the vectors $\mathbf{p}$ and $\mathbf{k}$, respectively. We solve Eq. (8) by converting the integral equation into a set of simultaneous non-linear equations. The range of integration used is $k=10^{-3}$ to $10^{3}$. The number of points per decade to carry out the integration was taken to be 31 which was found to be a sufficiently high number to obtain the accuracy with which the results have been quoted. In Fig. 1 we plot the mass function $M$ for $\alpha^{\prime}=1 / 4 \pi$ and various values of $T$ in the Landau gauge, i.e., $\xi=0$. With the increase of temperature, the generated mass decreases rapidly. The large $p^{2}$ behavior of the generated mass is depicted in Table I. We find that the mass function decreases as $1 / p^{2}$ in the asymptotic region of momentum.

The generated mass can be approximated by $M(0) \underline{18}$, 19], since for small $p, M(p)$ is rather constant. We draw it as a function of temperature in Fig. 22 in the Landau gauge. One can see a clear indication of the existence of a critical value $T_{c}$ of temperature above which there is no dynamical generation of mass. $T_{c}$ was found to be around 0.037915 . In order to see whether this critical value of the coupling can be obtained analytically, we analyze an approximated version of Eq. (8) in section [IC]

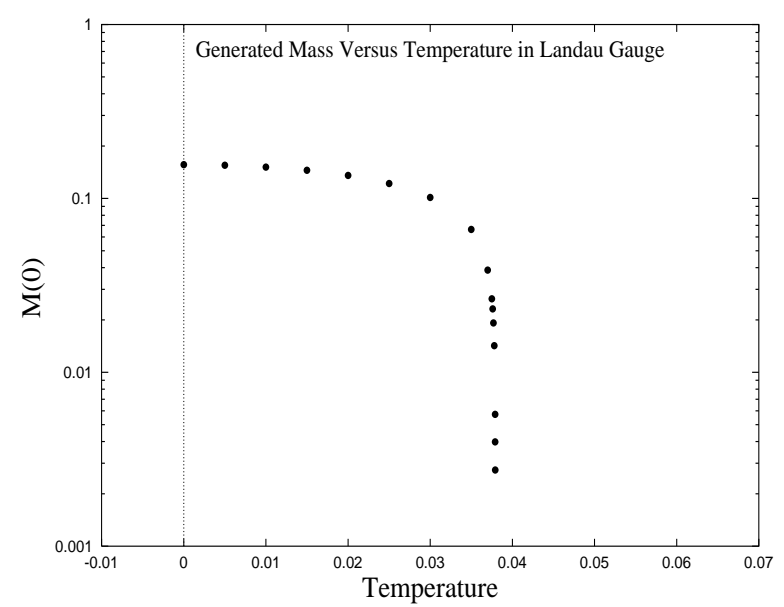

FIG. 2: The approximated generated mass $M(0)$ for the fermion for various values of temperature and $\alpha^{\prime}=1 / 4 \pi$ in the Landau gauge.

\section{B. Comparison with QED3 at zero temperature}

It is worth mentioning that at $T=0$, Eq. (8) is the same as the one in QED3 at zero temperature in the approximations of using the bare counterpart of the full vertex and setting $F=1$ (see Eq. (2.7) of Ref. [18]), with the only difference that the factor $2+\xi$ has been replaced with $3+\xi$. The origin of this only difference is the the fact that the trace algebra is carried out in different dimensions in the two cases. We now mention an important contrast with QED3 at zero temperature where under identical assumptions, equation for the mass function reads as

$$
M(p)=\frac{(2+\xi) \alpha}{\pi p} \int_{0}^{\infty} d k \frac{k M(k)}{k^{2}+M^{2}(k)} \ln \left|\frac{k+p}{k-p}\right|,
$$

This equation is scale invariant. The solution for an arbitrary value of $e^{2}$ can be obtained from $e^{2}=1$ in the following fashion:

$$
M\left(p, e^{2}\right)=e^{2} M\left(p / e^{2}, 1\right) .
$$

Therefore, if chiral symmetry breaking solution exists for one value of coupling it exists for all values of coupling. There is no critical value of $e^{2}$ or $\alpha$. A mathematically similar scaling law at finite temperature is

$$
M\left(p, e^{2}, T\right)=e^{2} M\left(p / e^{2}, 1, T / e^{2}\right) .
$$

We have seen that for $e^{2}=1$, there is a critical temperature, $T_{c}=0.037915$, which separates the chirally asymmetric solution from the symmetric one, in the Landau gauge. Therefore, we have

$$
M\left(p, 1, T_{>c}\right)=0 \quad \forall \quad p
$$

for all temperatures $T_{>c}$ above its critical value. What happens if we double the value of coupling $e^{2}$ ? The scaling law implies that

$$
M(p, 2, T)=2 M(p / 2,1, T / 2) .
$$




\begin{tabular}{|c|c|c|c|c|c|}
\hline$T$ & $p$ & $p^{2} M\left(p^{2}\right)$ & $T$ & $p$ & $p^{2} M\left(p^{2}\right)$ \\
\hline 0.0 & $\begin{array}{r}1000 \\
640.4 \\
304.699\end{array}$ & $\begin{array}{l}3.89920 \\
3.89940 \\
3.89947\end{array}$ & 0.037 & $\begin{array}{r}1000 \\
640.4 \\
304.699\end{array}$ & $\begin{array}{l}0.579892 \\
0.579921 \\
0.579931\end{array}$ \\
\hline 0.005 & $\begin{array}{r}1000 \\
640.4 \\
304.699\end{array}$ & $\begin{array}{l}3.84612 \\
3.84632 \\
3.84638\end{array}$ & 0.0375 & $\begin{array}{r}1000 \\
640.4 \\
304.699\end{array}$ & $\begin{array}{l}0.386170 \\
0.386189 \\
0.386196\end{array}$ \\
\hline 0.01 & $\begin{array}{r}1000 \\
640.4 \\
304.699\end{array}$ & $\begin{array}{l}3.68662 \\
3.68681 \\
3.68687\end{array}$ & 0.0376 & $\begin{array}{r}1000 \\
640.4 \\
304.699\end{array}$ & $\begin{array}{l}0.336004 \\
0.336021 \\
0.336027\end{array}$ \\
\hline 0.015 & $\begin{array}{r}1000 \\
640.4 \\
304.699\end{array}$ & $\begin{array}{l}3.41973 \\
3.41990 \\
3.41996\end{array}$ & 0.0377 & $\begin{array}{r}1000 \\
640.4 \\
304.699\end{array}$ & $\begin{array}{l}0.277625 \\
0.277640 \\
0.277644\end{array}$ \\
\hline 0.02 & $\begin{array}{r}1000 \\
640.4 \\
304.699\end{array}$ & $\begin{array}{l}3.04294 \\
3.04310 \\
3.04315\end{array}$ & 0.0378 & $\begin{array}{r}1000 \\
640.4 \\
304.699\end{array}$ & $\begin{array}{l}0.204152 \\
0.204163 \\
0.204166\end{array}$ \\
\hline 0.025 & $\begin{array}{r}1000 \\
640.4 \\
304.699\end{array}$ & $\begin{array}{l}2.54986 \\
2.54999 \\
2.55003\end{array}$ & 0.0379 & $\begin{array}{r}1000 \\
640.4 \\
304.699\end{array}$ & $\begin{array}{l}0.0818793 \\
0.0818835 \\
0.0818849\end{array}$ \\
\hline 0.03 & $\begin{array}{r}1000 \\
640.4 \\
304.699\end{array}$ & $\begin{array}{l}1.92211 \\
1.92220 \\
1.92224\end{array}$ & 0.03791 & $\begin{array}{r}1000 \\
640.4 \\
304.699\end{array}$ & $\begin{array}{l}0.0568280 \\
0.0568309 \\
0.0568319\end{array}$ \\
\hline 0.035 & $\begin{array}{r}1000 \\
640.4 \\
304.699\end{array}$ & $\begin{array}{l}1.08292 \\
1.08297 \\
1.08299\end{array}$ & 0.037915 & $\begin{array}{r}1000 \\
640.4 \\
304.699\end{array}$ & $\begin{array}{l}0.0391043 \\
0.0391063 \\
0.0391070\end{array}$ \\
\hline
\end{tabular}

TABLE 1. The large $p^{2}$ behavior of $M(p)$ for various values of temperature

\begin{tabular}{|c|c|c|c|c|c|}
\hline$T$ & $p$ & $p^{2} M\left(p^{2}\right)$ & $T$ & $p$ & $p^{2} M\left(p^{2}\right)$ \\
\hline 0.0 & $\begin{array}{r}1000 \\
640.4 \\
304.699\end{array}$ & $\begin{array}{l}2.41264 \\
2.41257 \\
2.41219\end{array}$ & 0.028 & $\begin{array}{r}1000 \\
640.4 \\
304.699\end{array}$ & $\begin{array}{l}0.402201 \\
0.402190 \\
0.402126\end{array}$ \\
\hline 0.005 & $\begin{array}{r}1000 \\
640.4 \\
304.699\end{array}$ & $\begin{array}{l}2.35491 \\
2.35485 \\
2.35448\end{array}$ & 0.0285 & $\begin{array}{r}1000 \\
640.4 \\
304.699\end{array}$ & $\begin{array}{l}0.268127 \\
0.268120 \\
0.268078\end{array}$ \\
\hline 0.01 & $\begin{array}{r}1000 \\
640.4 \\
304.699\end{array}$ & $\begin{array}{l}2.18138 \\
2.18132 \\
2.18098\end{array}$ & 0.0286 & $\begin{array}{r}1000 \\
640.4 \\
304.699\end{array}$ & $\begin{array}{l}0.234034 \\
0.234028 \\
0.233991\end{array}$ \\
\hline 0.015 & $\begin{array}{r}1000 \\
640.4 \\
304.699\end{array}$ & $\begin{array}{l}1.89032 \\
1.89027 \\
1.88997\end{array}$ & 0.0287 & $\begin{array}{r}1000 \\
640.4 \\
304.699\end{array}$ & $\begin{array}{l}0.194810 \\
0.194805 \\
0.194774\end{array}$ \\
\hline 0.02 & $\begin{array}{r}1000 \\
640.4 \\
304.699\end{array}$ & $\begin{array}{l}1.47509 \\
1.47505 \\
1.47482\end{array}$ & 0.0288 & $\begin{array}{r}1000 \\
640.4 \\
304.699\end{array}$ & $\begin{array}{l}0.146417 \\
0.146413 \\
0.146390\end{array}$ \\
\hline 0.025 & $\begin{array}{r}1000 \\
640.4 \\
304.699\end{array}$ & $\begin{array}{l}0.905525 \\
0.905501 \\
0.905357\end{array}$ & 0.0289 & $\begin{array}{r}1000 \\
640.4 \\
304.699\end{array}$ & $\begin{array}{l}0.0724110 \\
0.0724091 \\
0.0723976\end{array}$ \\
\hline 0.026 & $\begin{array}{r}1000 \\
640.4 \\
304.699\end{array}$ & $\begin{array}{l}0.762463 \\
0.762443 \\
0.762322\end{array}$ & 0.02891 & $\begin{array}{r}1000 \\
640.4 \\
304.699\end{array}$ & $\begin{array}{l}0.0602952 \\
0.0602936 \\
0.0602841\end{array}$ \\
\hline 0.027 & $\begin{array}{r}1000 \\
640.4 \\
304.699\end{array}$ & $\begin{array}{l}0.600565 \\
0.600549 \\
0.600454\end{array}$ & 0.02892 & $\begin{array}{r}1000 \\
640.4 \\
304.699\end{array}$ & $\begin{array}{l}0.0451881 \\
0.0451869 \\
0.0451798\end{array}$ \\
\hline
\end{tabular}

TABLE 2. The large $p^{2}$ behavior of $M(p)$ for various values of temperature for the approximated log. 


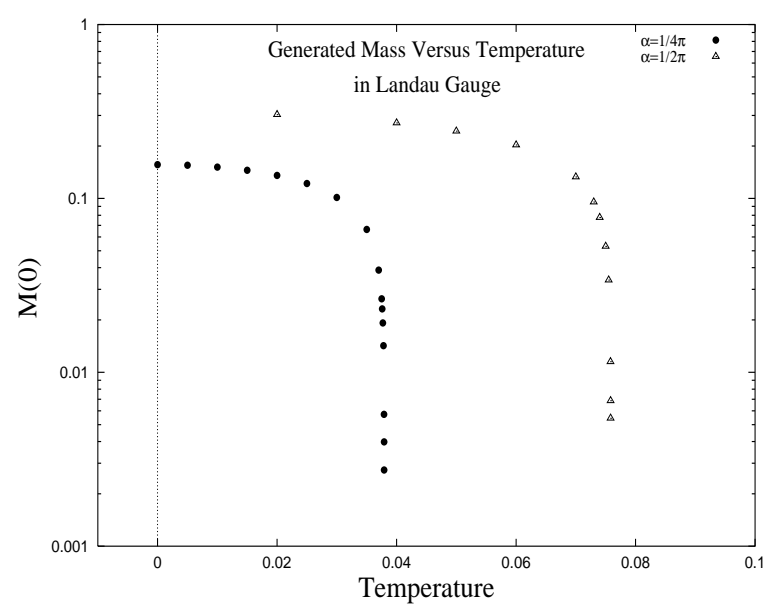

FIG. 3: The approximated generated mass $M(0)$ for the fermion for various values of temperature and two different values of coupling, $\alpha^{\prime}=1 / 4 \pi$ and $\alpha^{\prime}=1 / 2 \pi$ in the Landau gauge.

Let us substitute $T \rightarrow 2 T_{c}$ in this identity which gives

$$
M\left(p, 2,2 T_{c}\right)=2 M\left(p / 2,1, T_{c}\right) .
$$

Thus $M\left(p, 2,2 T_{>c}\right)=0$, i.e., the value of the critical coupling gets doubled by doubling the value of coupling $e^{2}$. To confirm this scaling law we study Eq. (8) for $\alpha^{\prime}=$ $1 / 2 \pi$ and find that the new value of critical temperature is indeed roughly 0.075825 . This is shown in Fig. 3.

\section{The equation with approximated log}

We can simplify Eq. (8) by employing the approximation [20],

$$
\ln \left|\frac{x+y}{x-y}\right| \equiv \frac{2 y}{x} \theta(x-y)+\frac{2 x}{y} \theta(y-x),
$$

which is a good approximation for $x<<y$ and $x>>y$. Moreover, for a small generated mass, we can also neglect the factor $M^{2}(k)$ in the denominator. Equation (8) can then be written as

$$
\begin{array}{r}
M(p)=\frac{(3+\xi) \alpha^{\prime}}{\pi} \int_{0}^{\infty} d k \frac{M(k)}{\pi^{2} T^{2}+k^{2}}[\theta(k-p) \\
\left.+\frac{k^{2}}{p^{2}} \theta(p-k)\right] .
\end{array}
$$

If we repeat the numerical analysis of the previous subsection, we find that the qualitative results do not undergo any drastic change. The mass function still lowers its height with temperature and above a critical value of the temperature (now 0.02893, see Fig. 5), only the trivial solution exists, i.e., $M(p)=0$. This behavior is shown in Fig. [1 in the Landau gauge. Moreover, the large momentum behavior of the mass function is again $1 / p^{2}$ as shown in Table II. This behavior for asymptotic values of momentum can be confirmed by converting Eq. (11) into a second order ordinary differential equation

$$
\frac{d}{d p}\left[p^{3} \frac{d M(p)}{d p}\right]+\frac{4(3+\xi) \alpha^{\prime} M(p)}{\pi}=0
$$

with boundary conditions

$$
p^{3} \frac{d M(p)}{d p}=\left.0\right|_{p=0} \quad \text { and } \quad M(p)=\left.0\right|_{p \rightarrow \infty},
$$

where we have dropped $\pi T$ in comparison with $p$. The solution of this equation is

$$
\begin{aligned}
M(p)=\frac{8(3+\xi) \alpha^{\prime}}{\pi p} & {\left[c_{1} J_{2}\left(4 \sqrt{\frac{(3+\xi) \alpha^{\prime}}{\pi p}}\right)\right.} \\
& \left.-c_{2} Y_{2}\left(4 \sqrt{\frac{(3+\xi) \alpha^{\prime}}{\pi p}}\right)\right]
\end{aligned}
$$

where $J(x)$ and $Y(x)$ are the Bessel functions of the first and second kind, respectively. The second boundary condition imposes $c_{2}=0$. As $J(1 / \sqrt{p}) \rightarrow 1 / p$ when $p \rightarrow \infty$, we conclude that $M(p) \rightarrow 1 / p^{2}$ in this limit, as found numerically. However, such an analytic treatment washes out the information about the effect of temperature and we cannot find the critical value of coupling. To retain the effect of temperature, note that the factor $\pi T$ in Eq. (11) serves as an infrared cutoff and therefore, we can rewrite this equation as

$$
\begin{aligned}
& M(p)=\frac{(3+\xi) \alpha^{\prime}}{\pi} \int_{\pi T}^{\infty} d k \frac{M(k)}{k^{2}} {[\theta(k-p)} \\
&\left.+\frac{k^{2}}{p^{2}} \theta(p-k)\right]
\end{aligned}
$$

The corresponding differential equation is exactly as we had obtained before. Only the first boundary condition changes to

$$
\left.p^{3} \frac{d M(p)}{d p}\right|_{p=\pi T}=0 .
$$

This boundary condition leads us to the following equation at the critical coupling

$$
J_{2}(4 a)+a\left[J_{1}(4 a)-J_{3}(4 a)\right]=0,
$$

where

$$
a=\sqrt{(3+\xi) \alpha^{\prime} / \pi^{2} T} .
$$

Note that $a$ being a constant [determined by Eq. (16)], we see that at the critical coupling, $\alpha^{\prime}$ and $T$ are proportional to each other, i.e., if we increase $\alpha^{\prime}$, the critical value of coupling will increase by the same factor. The first non-trivial zero of Eq. (16) yields $a=0.958$ 


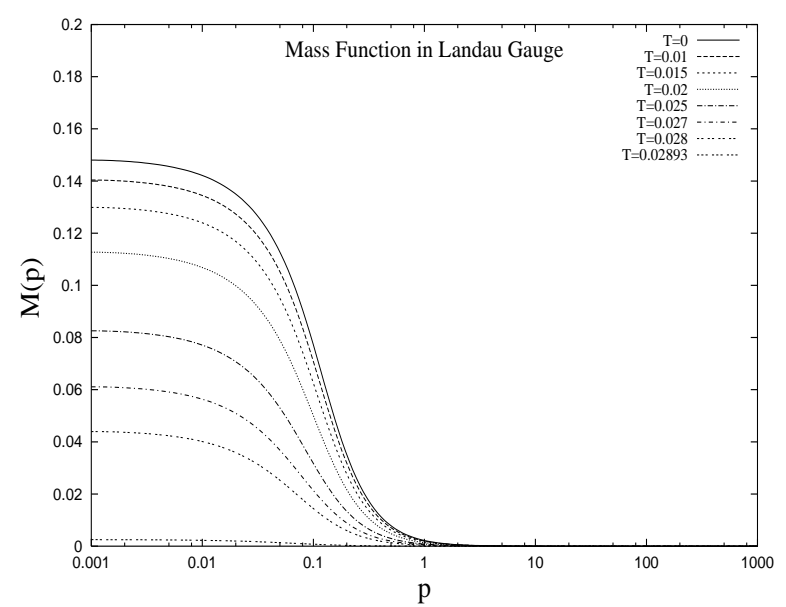

FIG. 4: The mass function $M(p)$ for various values of temperature and $\alpha=1 / 4 \pi$ for the approximated logarithm in the Landau gauge.

for $\alpha^{\prime}=1 / 4 \pi$. This implies $T=0.02636$ in the Landau gauge, which is within $10 \%$ of the actual numerical value.

What happens when we are in a gauge other than the Landau gauge? In principle, the critical values $\alpha_{c}$ and $T_{c}$ should be gauge independent because they trigger a phase transition. However, we do not expect it to be the case in the present work primarily because of the following reasons : (1) We assume the wave function renormalization $F(p)$ to be 1 . The studies at zero temperature indicate that the inclusion of the equation for $F(p)$ plays a crucial role in the partial restoration of gauge invariance, [18]. (2) The rainbow approximation neither respects the Ward-Green-Takahashi identity nor the Landau-Khalatnikov-Fradkin transformations, both being the consequences of gauge invariance. The gauge dependence of $T_{c}$ as a result of the numerical and analytical analyses has been plotted in Fig. (6). As another confirmation of the analytical result, Eq. (17), we find that in our numerical calculation, the critical temperature in an arbitrary covariant gauge, $T_{c}(\xi)$, is obtained from the one in the Landau gauge through the relation $T_{c}(\xi)=[(3+\xi) / 3] T_{c}$.

\section{SUMMING OVER MATSUBARA FREQUENCIES}

Here we want to show that, under some simplifications to analytically handle the expressions, the results obtained in the previous section appear to remain qualitatively unchanged, when one considers the contribution from Matsubara frequencies other than the lowest one. Let us stress that this point is by no means evident since

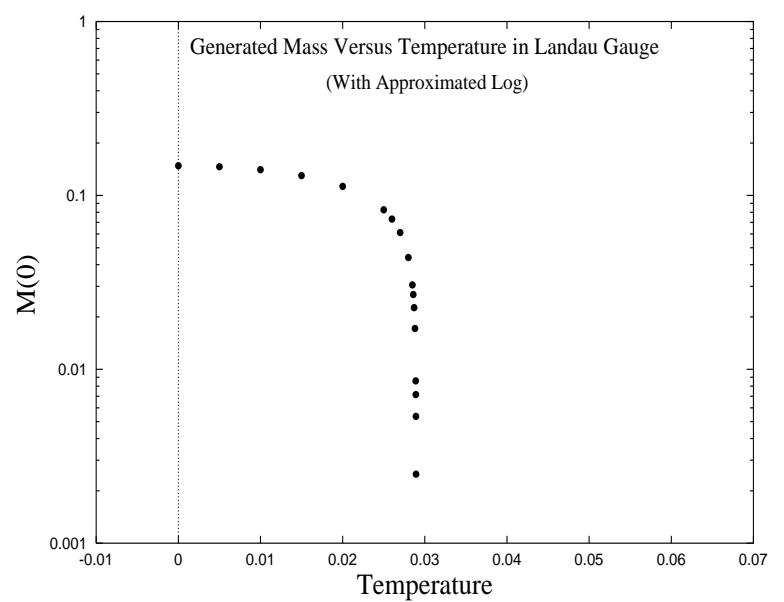

FIG. 5: The approximated generated mass $M(0)$ for the fermion for various values of temperature and $\alpha=1 / 4 \pi$ for the approximated logarithm in the Landau gauge.

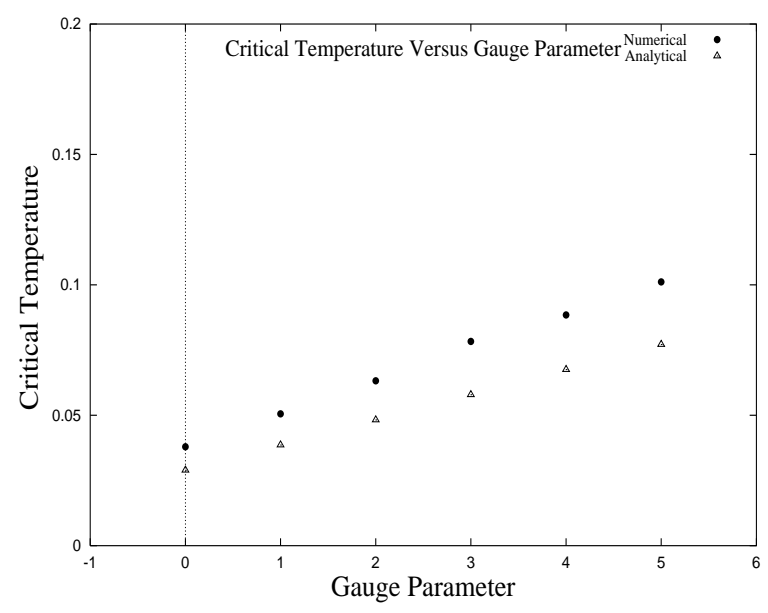

FIG. 6: Critical temperature $T_{c}$ versus the gauge parameter $\xi$. The upper and lower curves correspond to numerical and analytical results, respectively.

there are known examples in QED for which the contribution from the zeroth mode is exactly canceled by the contribution of the rest of the modes. One such example is provided by the one-loop fermion damping rate at finite temperature, which, as expected on physical grounds, when evaluated on the free mass shell vanishes due to the absence of phase space. This result can also be seen to arise as a consequence of the exact cancellation between the (divergent) contribution from the zeroth Matsubara mode and the rest of the modes 21].

Since we will be primarily interested in order of magnitude estimates, we will carry out some rough approximations aimed to preserve the possibility to analytically extract the behavior of the mass function in the critical region. The starting point is the sum over Matsubara 
frequencies indicated in Eq. (77) which we rewrite here as

$$
\begin{aligned}
I(i \omega, \mathbf{p}) & \equiv T \sum_{n} M(K) \\
& \times \frac{1}{\left[\omega_{n}^{2}+\mathbf{k}^{2}+M^{2}(K)\right]\left[\left(\omega_{n}-\omega\right)^{2}+|\mathbf{k}-\mathbf{p}|^{2}\right]} .
\end{aligned}
$$

As a first approximation, we neglect a possible dependence of $M(K)$ on the frequency, writing $M(K)=M(k)$. In this approximation, the sum in Eq. (18) can be computed using standard methods 22$]$ with the result

$$
\begin{aligned}
I(i \omega, \mathbf{p}) & =-\frac{1}{4 E_{1} E_{2}}\left\{\left[1+f\left(E_{2}\right)-\tilde{f}\left(E_{1}\right)\right]\right. \\
& \times\left(\frac{1}{i \omega-E_{1}-E_{2}}-\frac{1}{i \omega+E_{1}+E_{2}}\right) \\
& +\left[f\left(E_{2}\right)+\tilde{f}\left(E_{1}\right)\right] \\
& \left.\times\left(\frac{1}{i \omega-E_{1}+E_{2}}-\frac{1}{i \omega+E_{1}-E_{2}}\right)\right\},
\end{aligned}
$$

where $E_{1}=\sqrt{k^{2}+M^{2}(k)}, E_{2}=|\mathbf{k}-\mathbf{p}|$ and

$$
\begin{aligned}
& f(x)=\frac{1}{e^{x / T}-1} \\
& \tilde{f}(x)=\frac{1}{e^{x / T}+1}
\end{aligned}
$$

are the Bose-Einstein and Fermi-Dirac distributions, respectively. Equation (19) contains the usual vacuum and matter contributions present at finite temperature, these last weighed with the statistical distributions corresponding to the boson and fermion internal lines in the selfenergy diagram.

Having carried out the sum over frequencies, it is now possible to perform the analytical continuation of the function $I(i \omega, \mathbf{p})$ to real energy values. We consider the analytical continuation to the retarded function $I^{r}\left(p_{0}, \mathbf{p}\right)$. Although the function $I^{r}\left(p_{0}, \mathbf{p}\right)$ contains in principle an imaginary as well as a real part, in order to make the connection with the analysis in Sec. III] here we concentrate on the real part. As stressed in Ref. 7], attention must be paid to the imaginary part when considering the complete tensor structure of the fermion selfenergy and/or a more complete form of the gauge boson propagator. The real part of the function $I^{r}$ is defined as

$$
\operatorname{Re} I^{r}\left(p_{0}, \mathbf{p}\right)=\frac{1}{2}\left[I\left(i \omega \rightarrow p_{0}+i \epsilon\right)+I\left(i \omega \rightarrow p_{0}-i \epsilon\right)\right]
$$

and written explicitly as

$$
\operatorname{Re} I^{r}\left(p_{0}, \mathbf{p}\right)=-\frac{1}{4 E_{1} E_{2}}\left\{\left[1+f\left(E_{2}\right)-\tilde{f}\left(E_{1}\right)\right]\right.
$$

$$
\begin{aligned}
& \times \mathcal{P}\left(\frac{1}{p_{0}-E_{1}-E_{2}}-\frac{1}{p_{0}+E_{1}+E_{2}}\right) \\
& +\left[f\left(E_{2}\right)+\tilde{f}\left(E_{1}\right)\right] \\
& \left.\times \mathcal{P}\left(\frac{1}{p_{0}-E_{1}+E_{2}}-\frac{1}{p_{0}+E_{1}-E_{2}}\right)\right\},
\end{aligned}
$$

where $\mathcal{P}$ denotes principal part. It is now easy to see that the integral in Eq. (7) can now be expressed in Minkowski space in terms of the functions

$$
\begin{aligned}
I_{1}\left(p_{0}, p\right)= & \mathcal{P} \int \frac{d^{3} k}{(2 \pi)^{3}} \frac{M(k)}{4 E_{1} E_{2}} \\
& \left(\frac{1}{p_{0}+E_{1}+E_{2}}-\frac{1}{p_{0}-E_{1}-E_{2}}\right) \\
I_{2}\left(p_{0}, p\right)= & \mathcal{P} \int \frac{d^{3} k}{(2 \pi)^{3}} \frac{M(k)}{4 E_{1} E_{2}} f\left(E_{2}\right)\{ \\
& \left(\frac{1}{p_{0}+E_{1}+E_{2}}-\frac{1}{p_{0}-E_{1}-E_{2}}\right) \\
+ & \left.\left(\frac{1}{p_{0}-E_{1}+E_{2}}-\frac{1}{p_{0}+E_{1}-E_{2}}\right)\right\} \\
I_{3}\left(p_{0}, p\right)= & \mathcal{P} \int \frac{d^{3} k}{(2 \pi)^{3}} \frac{M(k)}{4 E_{1} E_{2}} \tilde{f}\left(E_{1}\right)\{ \\
& \left(\frac{1}{p_{0}-E_{1}-E_{2}}-\frac{1}{p_{0}+E_{1}+E_{2}}\right) \\
+ & \left.\left(\frac{1}{p_{0}+E_{1}-E_{2}}-\frac{1}{p_{0}-E_{1}+E_{2}}\right)\right\},
\end{aligned}
$$

which are thus written to separate the different vacuum and matter contributions. The choice equivalent to working with the lowest value of $i \omega$ is to take $p_{0}=0$. Also, since we are interested in looking for critical behavior, we neglect $M^{2}$ for which $E_{1} \rightarrow k$.

As a second simplification, let us ignore the angular dependence of both the distribution functions or the mass function. This can be thought of as considering the situation where $p \approx 0$. This is the region of interest for the present analysis since, as shown in the previous section, the small $p$ region is the relevant domain for the development of a non-trivial solution for $M$. The above simplification allows to analytically perform the angular integrations. Therefore, keeping in mind that we are interested in the situation where $p \rightarrow 0$, the set of Eqs. (23) becomes

$$
\begin{aligned}
& I_{1}(p)=\frac{1}{8 \pi^{2} p} \int_{0}^{\infty} d k M(k) \ln \left|\frac{p+2 k}{p-2 k}\right| \\
& I_{2}(p)=\frac{1}{8 \pi^{2} p} \int_{0}^{\infty} d k M(k) f(k) \ln \left|\frac{p+2 k}{p-2 k}\right| \\
& I_{3}(p)=-\frac{1}{8 \pi^{2} p} \int_{0}^{\infty} d k M(k) \tilde{f}(k) \ln \left|\frac{p+2 k}{p-2 k}\right| .
\end{aligned}
$$

Thus, in this limit, Eq. (7) can be written in Minkowski 
space as

$$
\begin{aligned}
M(p)= & \frac{(3+\xi) \alpha}{2 \pi p} \int_{0}^{\infty} d k M(k) \\
& {[1+f(k)-\tilde{f}(k)] \ln \left|\frac{p+2 k}{p-2 k}\right| . }
\end{aligned}
$$

We now use Eq. (10) to write Eq. (25) as

$$
\begin{aligned}
M(p)= & \frac{(3+\xi) \alpha}{2 \pi p}\{ \\
& \int_{0}^{p / 2} d k M(k)[1+f(k)-\tilde{f}(k)] \frac{4 k}{p} \\
+ & \left.\int_{p / 2}^{\infty} d k M(k)[1+f(k)-\tilde{f}(k)] \frac{p}{k}\right\} .
\end{aligned}
$$

Deriving Eq. (26) and multiplying by $p^{3}$, we obtain

$$
\begin{aligned}
p^{3} \frac{d M(p)}{d p} & =-\frac{4(3+\xi) \alpha}{\pi} \\
& \times \int_{0}^{p / 2} d k M(k)[1+f(k)-\tilde{f}(k)] k .
\end{aligned}
$$

For $p \rightarrow 0$, the integration region in Eq. (27) is that of small $k$. Notice that, in spite of the presence of the Bose-Einstein distribution, the integrand is infrared safe since

$$
[1+f(k)-\tilde{f}(k)] k \rightarrow T
$$

when $k \rightarrow 0$. We can incorporate this behavior by considering the dominant contribution to the integrand, namely, the one coming from the low $k$ expansion of the function $f(k) \approx T / k$ writing

$$
p^{3} \frac{d M(p)}{d p}=-\frac{4(3+\xi) \alpha}{\pi} T \int_{T}^{p / 2} d k M(k) .
$$

This requires the mass function $M$ to satisfy

$$
\left.p^{3} \frac{d M(p)}{d p}\right|_{p=2 T}=0
$$

and, by further deriving Eq. (29), to also satisfy the differential equation

$$
p^{3} \frac{d^{2} M(p)}{d p^{2}}+3 p^{2} \frac{d M(p)}{d p}+\frac{2(3+\xi) \alpha^{\prime}}{\pi} M(p)=0 .
$$

Equation (31) becomes identical to Eq. (12) after the substitution $T \rightarrow 2 T$. Thus, Eqs. (12) and (31) show that the scale for the onset of critical behavior can be obtained by considering only the lowest Matsubara mode in the analysis.

\section{CONCLUSIONS}

We have studied the SDE for the fermion propagator to find non-trivial solution for the mass function in qQED under the assumption that the fermion-photon vertex is bare, the full fermion propagator can be approximated by Eq. (5) and that only the zeroth frequency mode contributes dominantly. In order to stress the relation to the case of QED3, we have performed the analysis taking as independent parameters $\alpha^{\prime}=\alpha T$ and $T$. As expected, we observe that chiral symmetry is dynamically broken above a critical value of coupling $\alpha^{\prime}$ for a fixed temperature and is restored above a critical value of temperature while we hold the coupling constant. We also find that under simplifying assumptions, the result remains qualitatively unchanged by summing over all the frequency modes. Although not explicitly worked out here, the analysis can be also made considering $\alpha$ and $T$ as the independent parameters.

In order to clarify the meaning of the mass function found in this work, recall that in vacuum, the ultraviolet behavior of the theory is connected to the dynamical symmetry breaking phenomenon. The connection, in the case of theories with anomalies (e.g. the chiral anomaly) comes, on general grounds, through the impossibility of simultaneously regularizing the theory while preserving the classical symmetries. In fact, it is well known that in $\mathrm{qQED}$ in 4 dimensions at $T=0$, the generated mass function is proportional to the ultraviolet cut-off. However, the important point to keep in mind is that this happens because this cut-off is the only mass scale available in the theory.

The former does not mean that the ultraviolet divergences of the theory are essential for dynamical symmetry breaking. An example is provided by QED3 at $T=0$, where chiral symmetry is dynamically broken despite the fact that the theory is ultraviolet safe and super-renormalizable. In this case, the mass scale available is the dimensionful coupling.

The above remarks apply to the case of the theory in vacuum and keep being true at finite $T$ since the vacuum properties are left untouched by the temperature.

We should stress however that the mass function found in this work is thermal, i.e. it vanishes for $T=0$. This means that, unlike the vacuum case, the ultraviolet sector of the theory is not the one that provides the mass scale for dynamical symmetry breaking. This can be understood by noticing that any $T=0$ ultraviolet divergence is not present at finite $T$ since the thermal distributions cut off the integrals at large momentum. Thus, the mass scale is provided by the temperature and in fact our results show, for the case of qQED that we consider, that the generated mass function is proportional to $T$.

Criticality arises due to the existence of a dimensionless parameter. In QED4 at $T=0$, it is $\alpha$. In contrast, in QED3 at $T=0$, there is no such parameter and therefore, chiral symmetry is dynamically broken for all values of the coupling. In QED4 at finite $T$, taking $\alpha^{\prime}=\alpha T$ to 
be an independent parameter, the dimensionless parameter is provided by $\alpha^{\prime} / T$. Given the absence of ultraviolet divergences when considering only thermal effects, no additional scales come into play from the ultraviolet sector. The scaling law found is thus unaffected by the ultraviolet sector.

It will be interesting to see the impact on results if we include the wave function renormalization $F$ and the function $b$ in which case the analysis should account for the imaginary part of the self-energy [7]. It is also important to repeat the calculation accounting for a more complete fermion-photon vertex, studying the impact of this treatment on the gauge dependence of the critical values for chiral symmetry restoration. All this is for future.

\section{Acknowledgments}

AB gratefully acknowledges useful discussions with M.R. Pennington and the hospitality extended to him by the Institute of Particle Physics Phenomenology (IPPP), University of Durham, and the National Centre of Physics, Quaid-i-Azam University, during his visit there in the summer of 2002. Support for this work has been received in part by CIC under grant number 4.12, PAPIIT under grant number IN108001 and CONACyT under grants number 32395-E, 32279-E, 35792-E and 40025-F.
[1] A. Ayala and A. Bashir, Phys. Rev. D 64, 025015 (2001).

[2] K.-I. Kondo and K. Yoshida, Int. J. Mod. Phys. A 10, 199 (1995).

[3] M. Harada and A. Shibata, Phys. Rev. D 59, 014010 (1998).

[4] K. Fukazawa, T. Inagaki, S. Mukaigawa and T. Muta, Prog. Teo. Phys. 105, 979 (2001).

[5] T. Ikeda, Prog. Teo. Phys. 107, 403 (2002).

[6] Y. Fueki, H. Nakkagawa, H. Yokota and K. Yoshida, Prog. Teo. Phys. 107, 759 (2002).

[7] Y. Fueki, H. Nakkagawa, H. Yokota and K. Yoshida, Chiral phase transitions in QED at finite temperature: Dyson-Schwinger equation analysis in the real time HardThermal-Loop approximation, hep-ph/0212362

[8] N. Dorey and N.E. Mavromatos, Phys. Lett. B 266, 163 (1991).

[9] I.J.R. Aitchison, N. Dorey, M. Klein-Kreisler and N.E. Mavromatos, Phys. Lett. B 294, 91 (1992).

[10] I.J.R. Aitchison, and M. Klein-Kreisler, Phys. Rev. D 50, 1068 (1994).
[11] I.J.R. Aitchison, Z. Phys. C 67, 303 (1995).

[12] G. Triantaphyllou, Phys. Rev. D 58, 065006 (1998).

[13] G. Triantaphyllou, JHEP 9903, 020 (1999).

[14] D. Lee and G. Metikas, Int. J. Mod. Phys. A 14, 2921 (1999).

[15] P. Ginsparg, Nucl. Phys. B 170, 388 (1980).

[16] H.A. Weldon, Phys. Rev. D 26, 2789 (1982).

[17] A. Bashir and M.R. Pennington, Phys. Rev. D 50, 7679 (1994).

[18] A.Bashir, A. Huet and A. Raya, Phys. Rev. D 66, 025029 (2002).

[19] D. Atkinson, J.C.R. Bloch, V.P. Gusynin, M.R. Pennington and M. Reenders, Phys. Lett. B 329, 117 (1994).

[20] C.J. Burden and C.D. Roberts, Phys. Rev. D 44, 540 (1991).

[21] J.-P Blaizot and E. Iancu, Phys. Rev. D 55, 973 (1997).

[22] M. Le Bellac, Thermal Field Theory (Cambridge University Press, Cambridge, England, 1996). 